\title{
Contexto universitario, profesores y estudiantes: vínculos y éxito académico
}

\author{
Jorge Humberto Guanin-Fajardo ${ }^{1}$ (D) \\ Jorge Casillas Barranquero ${ }^{2}$ (D) \\ ${ }^{1}$ Universidad Técnica Estatal de Quevedo (UTEQ), Ecuador; ${ }^{2}$ Universidad de Granada (UGR), España
}

Resumen. La promoción de una educación de calidad en las instituciones de enseñanza superior promueve la autoeficacia. El objetivo del trabajo está dirigido al análisis de las características del profesorado y al éxito académico de los estudiantes al final del primer año en el contexto universitario. La población estudiada fue de 6690 estudiantes y 256 profesores, el conjunto de datos lo conforman 15 variables entre numéricas y categóricas. Se utilizó estadística descriptiva, métricas diseñadas para evaluar datos significativos y técnicas avanzadas de visualización. Los resultados revelaron el perfil esencial de los profesores experimentados y maduros, tanto en la enseñanza como en los grupos de edad. Los profesores experimentados que participaron en la enseñanza en un porcentaje superior al $66 \%$, influyeron con un $72 \%$ de certeza en el éxito académico del alumnado. A corto plazo, los profesores noveles cuya tasa de participación fue del 33\% mostraron un efecto positivo. A largo plazo, los estudiantes cambiaron (8\%) o abandonaron (59\%) la titulación universitaria. La utilidad de estos resultados aporta sugerencias para una enseñanza significativa y oportuna, siempre que la distribución del profesorado experimentado y maduro corresponda con dos o tres tercios del total de profesores en el primer año de la titulación universitaria.

Palabras clave: éxito académico; contexto universitario; análisis educativo; técnicas de visualización.

Contexto universitário, professores e alunos: vínculos e sucesso acadêmico

Resumo. A promoção da educação de qualidade nas instituições de ensino superior promove a autoeficácia. O objetivo do trabalho foi direcionado para a análise das características do corpo docente e do sucesso acadêmico dos alunos ao final do primeiro ano no contexto universitário. A população estudada foi de 6.690 alunos(as) e 256 professores(as), o conjunto de dados contou com 15 variáveis entre numéricas e categóricas. Foram utilizadas estatística descritiva, métricas desenhadas para avaliar dados significativos e técnicas avançadas de visualização. Os resultados revelaram o perfil essencial de professores experientes e maduros, tanto na docência quanto nas faixas etárias. Os professores experientes que participaram da docência em percentual superior a 66\%, influenciaram com $72 \%$ de certeza no sucesso acadêmico dos alunos. No curto prazo, os professores iniciantes, cuja taxa de participação foi de 33\%, mostraram um efeito positivo. No longo prazo, os alunos(as) mudaram (8\%) ou abandonaram (59\%) o curso universitário. A utilidade desses resultados oferece sugestões para um ensino significativo e oportuno, desde que a distribuição de professores experientes e maduros corresponda a dois ou três terços do total de professores do primeiro ano do curso universitário.

Palavras-chave: sucesso acadêmico; contexto universitário; análise educacional; técnicas de visualização.

\section{University context, teachers and students: links and academic success}

Abstract. The promotion of quality education in higher education institutions promotes self-efficacy. The objective of the work was directed to the analysis of the characteristics of the faculty and the academic success of students at the end of the first year in the university context. The population studied was 6690 students and 256 professors, the data set had 15 variables between numerical and categorical. Descriptive statistics, metrics designed to evaluate meaningful data and advanced visualization techniques were used. The results revealed the essential profile of experienced and mature teachers, both in teaching and age groups. Experienced teachers who participated in teaching at a rate of more than $66 \%$, influenced with $72 \%$ certainty the academic success of the student body. In the short term, novice teachers whose participation rate was 33\% showed a positive effect. In the long term, students changed (8\%) or dropped out (59\%) of the university degree. The usefulness of these results provides suggestions for meaningful and timely teaching, provided that the distribution of experienced and mature faculty corresponds to two to three thirds of the total number of first-year faculty in the university degree program.

Keywords: academic success; university context; educational analysis; visualization techniques. 


\section{Introducción}

La educación, como parte fundamental del progreso de las sociedades y economías modernas impulsadas por la innovación y el desarrollo científico, nunca ha sido tan omnipresente como ahora (Marginson, 2014). Por ello, las instituciones de educación superior se han dedicado a promover la buena educación por diversas razones. En primer lugar, porque les interesa demostrar que son proveedores fiables de una educación de buena calidad, al tiempo que sirven a múltiples partes interesadas con diferentes expectativas (grados universitarios). En segundo lugar, porque deben responder a la creciente demanda de una educación significativa y oportuna. Por último, porque los resultados de la investigación son insuficientes para mantener la reputación de las instituciones de enseñanza superior, por lo que es esencial equilibrar los resultados de la enseñanza y el aprendizaje con los de la investigación (Nasser-Abu, 2017). En este sentido, los investigadores educativos han logrado crear herramientas tecnológicas, planes pedagógicos y/o curriculares, modelos predictivos, etc., que suministren a los responsables académicos recursos para usar estrategias controladas y proporcionadas que retengan al alumnado en el grado universitario inicial (Araque et al., 2009; Mishra y Sahoo, 2016; Van den Berg y Hofman, 2005). Desde de este punto de vista, el contexto universitario gestionado por las instituciones de educación superior es diverso, por ello, la aplicación de políticas y normas que regulan su actividad facilitan el progreso de las instituciones. No obstante, su diversidad más extendida y compleja se localiza en el recurso humano, de servicio, de infraestructura, económico, tecnológico, entre otros. Esta complejidad, trasciende en el grado de impacto y aceptación en la sociedad moderna, de hecho, el prestigio se puede alcanzar ajustando los recursos del contexto universitario. Para lograr el perfil de aceptación institucional, se proyecta estudiar el contexto universitario basado en dos recursos humanos esenciales: profesor y estudiante.

Partiendo de este punto, se encuentran cinco tipos de investigaciones en el ámbito educativo que han permitido descubrir información reveladora en datos académicos. En primer lugar, está el análisis de redes sociales que estudia diferentes iteraciones e implicaciones generales (Mishra, 2020; Trolian et al., 2021). En segundo lugar, los estudios longitudinales destinados a mejorar los resultados de los estudiantes, por ejemplo, (Amida et al., 2020; Souchon et al., 2020). En tercer lugar, el estudio del análisis factorial para investigar los factores ocultos en las interacciones entre alumnos y profesores (Le et al., 2017). En cuarto lugar, el meta-análisis examina características relacionadas con la implementación de estrategias para el aprendizaje del rendimiento académico de los estudiantes (De Boer et al., 2014). Por último, la minería de datos que explora mediante dos técnicas el descubrimiento de conocimiento: (i) técnicas no supervisadas, divididas en dos sub-técnicas. a) agrupamiento basado en estudios de distancia o similitud de vectores (Vo et al., 2016). b) reglas de asociación, para descubrir los hechos que ocurren dentro de los datos (Aleksandrova y Parusheva, 2019; Alyahyan y Düştegör, 2020; Guanin-Fajardo et al., 2019; Sanvitha Kasthuriarachchi et al., 2018). (ii) técnicas supervisadas, que predicen los datos mediante una variable dependiente (Shetu et al., 2021). La convergencia de las investigaciones ha coincidido en la flexibilidad de la mejora de los resultados académicos, la calidad de las relaciones de la comunidad universitaria, la mejora de los canales de comunicación, la buena enseñanza, la proyección de los objetivos, entre otros. 


\subsection{Objetivo del estudio}

El objetivo principal del trabajo está centrado en analizar las variables que determinan el éxito académico del alumnado y profesorado dentro del contexto universitario. Por consiguiente, se examinará el vínculo existente entre estudiantes y profesores, de modo que, se han planteado las siguientes preguntas de investigación:

- ¿Cuáles son los factores del profesorado que han influido en el éxito académico del estudiante?

- En el contexto universitario, ¿qué tipo de compatibilidad del profesorado se corresponde con el éxito académico de los estudiantes?

Para ello, se examinan los datos en profundidad para extraer información útil y relevante sobre las características del docente. En este punto, el estudio se divide en tres etapas: (i) recuperación de datos del sistema informático; (ii) análisis y aplicación de procedimientos para extraer datos significativos mediante las métricas propuestas; y, (iii) presentación de los principales resultados mediante técnicas de visualización. El análisis de datos propuesto pretende obtener información significativa sobre los factores del profesorado y el impacto en el alumnado para completar la titulación universitaria al finalizar el primer año. El presente trabajo está orientado a motivar la toma de decisiones y ser precursor de futuros estudios de análisis de datos exhaustivos para comprobar posibles teorías. Para ello, se creó una biblioteca personalizada de análisis de datos utilizando el programa estadístico $R$, que es un lenguaje de libre acceso para la computación estadística y que proporciona una amplia variedad de técnicas estadísticas y gráficas: modelización lineal y no lineal, pruebas estadísticas, clasificación, agrupación, entre otras (R CoreTeam, 2019).

\section{Trabajos relacionados}

Las instituciones de educación superior centran sus esfuerzos en el desarrollo de habilidades o atributos curriculares para que los estudiantes tengan una alta probabilidad de éxito académico (Leal et al., 2016). Partiendo de este punto, la oferta académica y el alcance de los servicios de las instituciones de educación superior son cruciales para el éxito académico. A vista del trabajo de (Respondek et al., 2017), la conceptualización del éxito académico se perfila en dos partes: (i) baja intención de abandono; y, (ii) alto rendimiento académico. Ambas debidamente sincronizadas responden positivamente al éxito académico del alumnado. De ahí que, la armonización del rendimiento académico y la motivación del alumnado descansen significativamente en el profesorado. Esta relación, trasciende por la claridad percibida en la enseñanza del profesor, capacidad y apoyo, así como en la implicación del nivel de satisfacción y la experiencia universitaria (Livengood, 1992; Pascarella et al., 1996).

\subsection{Contexto universitario}

El contexto universitario asocia múltiples factores para fortalecer e influir en el éxito académico de los estudiantes (Struyven et al., 2003). Siguiendo el trabajo de Winterer et al. (2020), los autores sugieren prácticas y normas que han facilitado el éxito académico: (i) la mejora del clima estudiantil; (ii) la calidad del acceso, el conocimiento de los estudiantes y el servicio de orientación (Korobova y Starobin, 2015); y, (iii) el aumento y la mejora de la calidad de los programas y servicios de asistencia 
académica (Kara et al., 2020). Estas prácticas estimulan la calidad en las relaciones de la comunidad universitaria y promueven espacios socialmente aceptables (Pineda et al., 2014). La incorporación del aprendizaje basado en proyectos dentro del plan de estudios también se ha considerado un enfoque exitoso (Konrad et al., 2021; Leal et al., 2016). De hecho, este aprendizaje expone numerosos conocimientos útiles, además de una contribución al crecimiento profesional y al aprendizaje de meta-habilidades (Salminen-Tuomaala y Koskela, 2020). Por otro lado, es difícil no valorar la tutoría como un factor vinculado al éxito académico y al liderazgo de los estudiantes durante la fase universitaria, dado que ha estado empíricamente relacionado con el desarrollo profesional (Campbell et al, 2012; Cunha et al., 2018; Jacobi, 1991). En líneas generales, en cualquier contexto universitario el profesorado tuvo un alto porcentaje de participación en la formación del estudiantado, es decir, todo el proceso de enseñanza y desarrollo profesional del estudiantado estriba en el profesorado.

\subsection{El éxito académico y el profesorado}

En cuanto a las expectativas del éxito académico del estudiantado subyace el desánimo como consecuencia del tipo y el contexto de las iteraciones con el profesorado. La importancia de una buena calidad por parte del profesorado y su influencia está bien vista por el alumnado (Cho et al., 2011). Esto fue reforzado por Lizzio et al. (2002), que dentro de sus hallazgos, afirmaron que la buena enseñanza tenía un efecto positivo en los resultados académicos y que estaba fuertemente asociada con el éxito académico. Sustancialmente, la calidad del profesorado es un precursor del éxito académico en el estudiante, ya que la perspectiva de terminar los estudios se fortalece. De esta manera, han surgido muchas investigaciones para conocer los factores influyentes entre profesores y el éxito académico del estudiantado (Chickering y Gamson, 1987; Crispet al., 2015; Tinto, 1975; Walder, 2017). No obstante, se han encontrado también trabajos que destacan la influencia negativa (Glogowska et al., 2007; Young et al., 2007). En cierto modo, el sistema educativo busca constantemente fomentar canales de comunicación profesor-alumno y alumno-alumno que es el eslabón clave para lograr el éxito académico. De alguna manera, la colaboración y participación mutua entre ellos genera un ambiente de confianza y cooperación para lograr los objetivos proyectados (Abarca et al., 2015). De ahí que la consolidación de la fluidez de los distintos canales de comunicación hayan promovido iteraciones que han impulsado al alumnado a alcanzar el éxito académico (Chickering y Gamson, 1987; Mishra, 2020; Trolian et al., 2021; Winterer et al., 2020). Al mismo tiempo, este hecho tiene una consecuencia importante, ya que pueden beneficiarse de oportunidades fuera del aula que enfatizan el valor del trabajo intelectual y el apoyo académico (Nagda et al. 1998). De todo lo que se ha dicho sobre la eficacia de la comunicación e iteración entre el profesorado y alumnado, otro punto a tener en cuenta está relacionado con los factores y la calidad del profesorado. De ahí que, por ejemplo han surgido estudios sobre: la edad (Campbell et al., 2005), la titulación académica y la experiencia docente (Angervall, 2018; Darling-Hammond, 2000; Jepsen, 2005; Korhonen y Törmä, 2016) que son tres factores que han influido en el éxito académico del estudiantado. 


\section{Metodología}

\subsection{Contexto}

Para realizar el estudio se ha considerado una Institución de Educación Superior (IES), las políticas universitarias de la misma y el cumplimiento de los requisitos mínimos que permiten al estudiantado superar cada año académico. La modalidad de estudio de la IES es presencial y el ciclo académico consta de dos semestres, el estudiantado debe superar ambos semestres para pasar al curso inmediato superior, debiendo alcanzar la nota mínima exigida en cada asignatura (7, en una escala de 0 a 10). La variable dependiente que determina el estado académico del estudiante se ha definido en función de las actividades académicas de cada semestre y curso. Siendo el estado Superado cuando todos los cursos de la titulación inicial han sido superados. Abandona, cuando a partir de la primera matrícula existe ausencia de actividades académicas; y, Cambio, cuando se superen los cursos de una titulación diferente a la inicial. La IES está localizada geográficamente en el cantón Quevedo, Los Ríos, Ecuador.

\subsection{Recolección de datos}

El proceso de recolección de datos se realizó extrayendo la información del sistema informático de la IES, que almacena toda la actividad académica entre profesores y alumnos. Obteniendo, en primer lugar la información derivada del proceso evaluativo desarrollado a lo largo del grado académico entre ambos. Después, el Departamento de Recursos Humanos aportó información cualitativa del profesorado, que se agregó y relacionó para formar el conjunto de datos final. Por último, la filtración de los datos fue establecida en seis periodos académicos entre el primer y el quinto año de las titulaciones universitarias ofertadas. A su vez, las políticas de protección de datos fueron aplicadas de acuerdo con los criterios de la Dirección de Planificación Académica de la Universidad que fue quien aprobó la recolección de datos.

\subsection{Datos}

El conjunto de datos original tuvo 6.690 registros y 15 variables categóricas y numéricas (véase el anexo 1). En este estudio, se ha utilizado como población a los estudiantes que estuvieron matriculados en el primer año y que finalizaron el grado académico. Además, se incorporó la población total de profesores que fue de 286 , incluyendo profesores titulares, agregados y ocasionales.

\subsection{Preparación de los datos}

Esta es una etapa importante del estudio, ya que es fundamental contar con datos claros y de buena calidad. Para ello, se han aplicado correctivos para los valores ausentes, ya que es común en problemas reales la omisión involuntaria de transcripción o recuperación automática de datos que se quedan sin valores, después, a estos datos se ha dado uniformidad según la ecuación 3. 


\subsection{Métricas}

En este estudio se han propuesto seis tipos de métricas para transformar y evaluar la calidad de los datos. La ecuación 1 ha ponderado las titulaciones del profesorado, donde $\mathrm{x}=$ cantidad de profesores con grado académico (licenciatura, ingeniería, biología, etc.); $y$ = cantidad de profesores con títulos de máster; $z$ = cantidad de profesores con títulos de doctorado; $y$, por último, $n=$ total de profesores que han impartido clases en el curso académico.

$$
w=\left[\left(\frac{x}{n} * 0.10\right)+\left(\frac{y}{n} * 0.30\right)+\left(\frac{z}{n} * 0.60\right)\right]
$$

(Ecuación 1)

La ecuación 2, es la métrica lift que comúnmente se utiliza en minería de datos, ya que sirve para mejorar la confianza de las reglas de asociación, donde tanto $x$ como $y$ son elementos del conjunto de datos (Brin et al., 1997). La ecuación se define como:

$$
\operatorname{Lift}(X \rightarrow Y)=\frac{\text { Confidence }(X \rightarrow Y)}{\operatorname{Support}(Y)}
$$

(Ecuación 2)

Donde confidence $=\frac{\operatorname{Support}(X \cup Y)}{\operatorname{Support}(Y)}, \mathrm{y}, \operatorname{Support}(Y)$ es definido como la proporción de transacciones en el conjunto de datos que contiene Y. Por otra parte, en la ecuación 3 se propone la fórmula que ha servido para obtener datos uniformes. Donde $Z_{i}$ es la variable normalizada [0-1], siendo $x_{\min }$ y $x_{\max }$ el valor mínimo y máximo de la variable respectivamente.

$$
Z_{i}=\frac{x_{i}-x_{\min }}{x_{\max }-x_{\min }}
$$

(Ecuación 3)

Las ecuaciones 4, 5 y 6 fueron planteadas para un análisis profundo de la información, donde se analizó el desorden de los datos (entropía de la información), la ganancia de información y la tasa de ganancia (Romanski y Kotthoff, 2016). Con estas ecuaciones se han obtenido cuantitativamente el comportamiento de las variables.

$$
\text { Symmetrical.uncertainty }=2 \frac{H(\text { Class })+H(\text { Attribute })-H(\text { Class }, \text { Attribute })}{H(\text { Attribute })+H(\text { Class })} \quad \text { (Ecuación 4) }
$$$$
\text { Information.gain }=H(\text { Class })+H(\text { Attribute })-H(\text { Class, Attribute })
$$

(Ecuación 5)

$$
\text { Gain.ratio }=\frac{H(\text { Class })+H(\text { Attribute })-H(\text { Class }, \text { Attribute })}{H(\text { Attribute })}
$$

(Ecuación 6)

\subsection{Procedimiento}

Para lograr el objetivo propuesto en este trabajo, se ha creado una librería personalizada con el programa estadístico R; esta librería tiene varias funciones para el procesamiento, análisis de datos y visualización de resultados. Para ello, se tienen considerados seis pasos: Primero, sustituir los datos ausentes en el conjunto de datos por datos aproximados utilizando medidas estadísticas de posicionamiento central (media, mediana y moda) (Breiman, 2001), según el tipo de variable. Segundo, normalizar 
las variables para obtener datos homogéneos [0-1]. Tercero, estudiar inicialmente las variables a través del cluster jerárquico, que ha servido para concentrar las variables según el grado de similitud utilizando para ello la distancia euclidiana. Cuarto, analizar el comportamiento de las variables con las métricas: curtosis, asimetría, incertidumbre, ratio de ganancia y ganancia de información, esto, para el filtrado de las tres variables principales que servirán para el análisis. Quinto, categorizar las variables para calcular la tabla de contingencia, con el fin de obtener las proporciones de las categorías frente a la variable dependiente (Abandona, Cambio, Superado), además de calcular la métrica lift (ecuación 2) para obtener el grado de confianza entre los datos encontrados. Por último, conseguir proyectar los resultados significativos en gráficos para mejorar la comprensión de los hallazgos obtenidos.

\section{Resultados}

En esta sección se presentan los principales resultados obtenidos a través del análisis profundo de los datos, que son las respuestas resultantes a las preguntas iniciales de este trabajo. Para ello, se utilizaron las métricas propuestas con el fin de conocer el comportamiento de las variables. Así, como las técnicas estadísticas estudiadas, que son: por un lado, la forma y distribución general de los datos y, por otro, la relación que ha existido entre ellos.

\subsection{Estudio exploratorio}

Como punto de partida para la exploración de los datos, se ha evaluado la información desde dos perspectivas. La primera, fue crear el conglomerado jerárquico, calculando la similitud entre las variables mediante la distancia Euclidiana que ha ayudado a comprender los grupos de variables. En consonancia con lo anterior, se muestra en la columna 3 de la Tabla 1, la media de participación del profesorado respecto a sus edades ha sido superior en Edad2 e inferior en Edad3 y Edad1. Sin embargo, la desviación típica (columna 2) en Edad1 fue mayor, lo que indica que también hubo una participación significativa de los profesores en este grupo de edad. Por otra parte, la media de experiencia docente y titulación docente fue de 16 años y 0.26 respectivamente. La razón fundamental de estos valores fue dada por la participación en mayor cantidad de profesores del grupo Edad2.

Tabla 1. Métricas de centralidad y tendencia de las variables independientes*.

\begin{tabular}{lllll}
\hline \multicolumn{1}{c}{ Variables } & Desviación típica & Media & Mínimo & Máximo \\
\hline Facultad & & & 1 & 5 \\
GradoUniversitario & & 1 & 22 \\
Edad1 & 2.9127 & 2 & 0 & 15 \\
Edad2 & 2.3867 & 5 & 0 & 11 \\
Edad3 & 1.8906 & 2 & 0 & 10 \\
ExperienciaDocente & 4.2912 & 16 & 4 & 26 \\
MediaEdadProfesor & 6.2228 & 53 & 35 & 64 \\
TitulaciónDocente & 0.0943 & 0.26 & 0.1 & 0.6
\end{tabular}




\begin{tabular}{lllll}
\hline \multicolumn{1}{c}{ Variables } & Desviación típica & Media & Mínimo & \multicolumn{1}{c}{ Máximo } \\
\hline MediaNotas & 1.3044 & 7.33 & 0.03 & 10 \\
MediaAsistencia & 6.5976 & 97 & 21 & 100 \\
TiempoSupera & 0.4911 & 1 & 1 & 5 \\
TasaSuperacion & 0.2440 & 1.05 & 0.052 & 2.708 \\
CuentaGrados & 0.2490 & 0 & 0 & 2 \\
CambioGrado & & & 1 & 2
\end{tabular}

*Las variables categóricas tienen valores vacíos en la columna de desviación típica y media.

Fuente: Elaboración propia

Y como segunda perspectiva el conglomerado jerárquico fue dividido en cuatro grupos de variables según el grado de similitud (Gráfico 1). Para ello, fueron calculadas y agrupadas las variables según la distancia euclidiana. Es decir, se mide la distancia que hay entre una variable y otra, aquí han destacado dos grupos relacionados con el profesorado (G2 y G4). El rendimiento académico del alumnado estuvo agrupado en G1. Por último, G3 estuvo compuesto con variables vinculadas al rendimiento académico (Tiempo superación, cuenta grados), profesorado (Edad2) y titulación académica (Facultad, grado universitario).

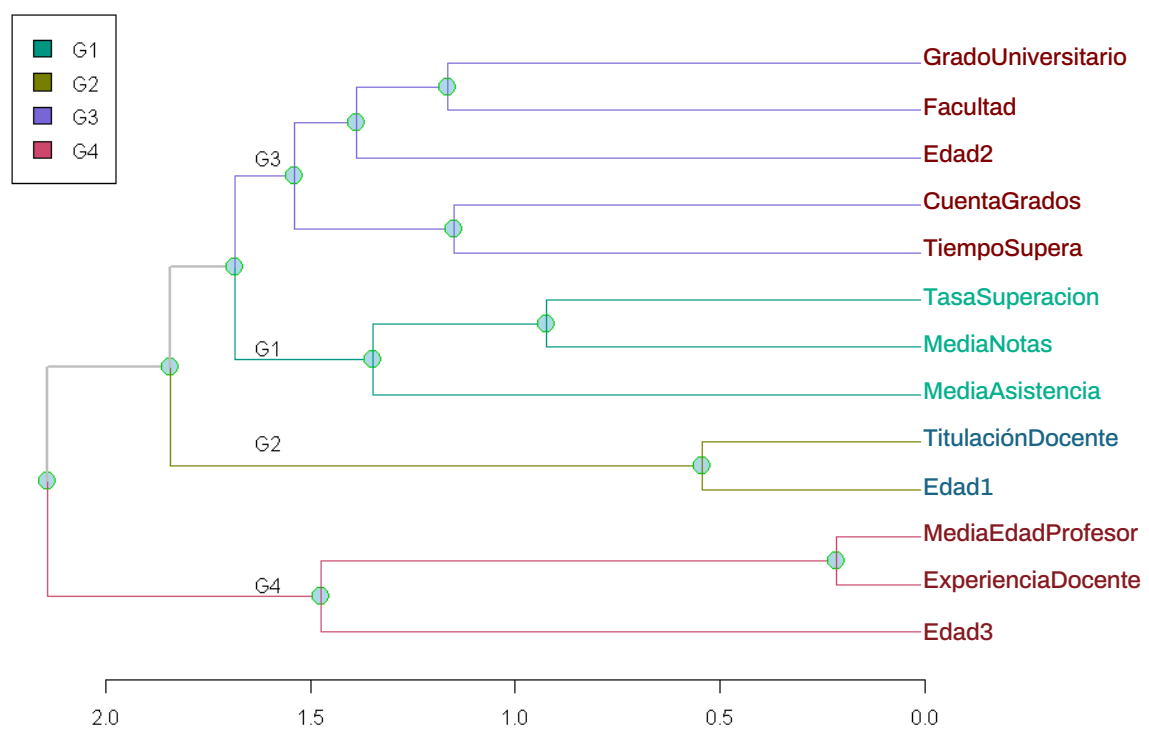

Gráfico 1. En el conglomerado jerárquico se han diferenciado cuatro subgrupos de variables, Los subgrupos G2 y G4 se han vinculado con las características del profesorado, G1 fue asociado con el rendimiento académico, y G3 ha implicado una mezcla de variables entre estudiantado y profesorado.

Fuente: Elaboración propia. 


\subsection{Análisis de los datos relacionados con los factores del profesorado}

En este apartado, se examina en profundidad las variables que han sido vinculadas al profesorado. Para ello, en el diagrama de Sankey se visualiza la carga y distribución de datos entre las variables. A su vez, en la Tabla 2 se han estudiado las variables con las métricas: asimetría, curtosis, incertidumbre, ganancia de información y tasa de ganancia. De acuerdo con este análisis, se filtró las tres primeras variables para examinar la incidencia del profesorado con los alumnos que han tenido éxito académico.

Tabla 2. Estudio profundo de variables relacionadas al profesorado, ordenadas según el nivel de incertidumbre (Uncertainty).

\begin{tabular}{lrrccc}
\hline \multicolumn{1}{c}{ Variables } & Asimetría & Curtosis & Uncertainty* & InforGain & GainRatio \\
\hline Edad3 & 1,0079 & 0,6380 & 0,0229 & 0,0213 & 0,0218 \\
Edad2 & 0,0790 & $-0,6948$ & 0,0294 & 0,0333 & 0,0241 \\
ExperienciaDocente & 0,2699 & 0,0489 & 0,0486 & 0,0557 & 0,0394 \\
MediaEdadPofesorado & 0,4500 & $-0,2568$ & 0,0609 & 0,0703 & 0,0492 \\
TitulacionDocente & 0,8466 & 0,7942 & 0,0701 & 0,0813 & 0,0564 \\
Edad1 & 1,7087 & 2,8320 & 0,0742 & 0,0662 & 0,0731 \\
* Orden ascendente & & & & & \\
\hline
\end{tabular}

Fuente: Elaboración propia

Las variables asociadas al profesorado, por ejemplo, la asimetría de Edad3 fue 1,0079, donde se evidenció inicialmente que pocos profesores de ese rango de edad participan en las clases del alumnado. Sin embargo, lo contrario ha sucedido con la Edad1 con asimetría de 1,7087, que mostró una mayor presencia de profesores en este rango de edad. Por otra parte, Edad2 y ExperienciaDocente presentaron también poca participación de profesores.

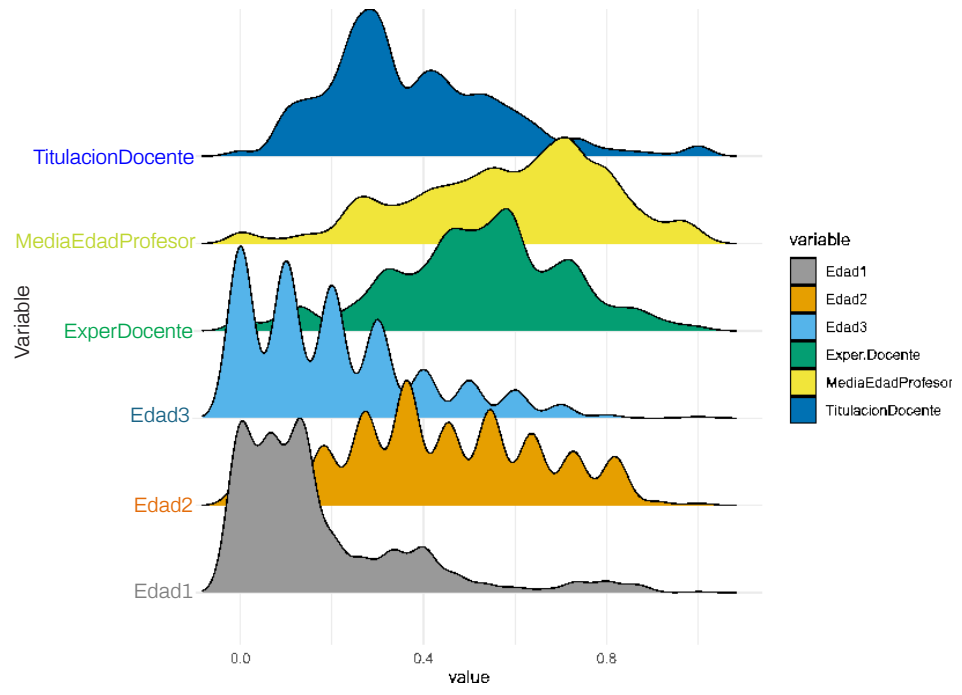

Gráfico 2. Densidad de variables relacionadas con las características del profesorado. La asimetría de las variables Exper.Docente y MediaEdadProfesor son mostradas hacia la derecha, mientras que el resto de variables hacia la izquierda.

Fuente: Elaboración propia. 
En el Gráfico 2, se condensan los resultados de la Tabla 2, en concreto lo relacionado con la asimetría y curtósis, donde gráficamente se ha valorado la distribución y tendencia de los datos. Por otra parte, en el diagrama de Sankey (Gráfico 3) llama la atención seis grupos de datos: primero, Edad1[0-0,33) ha mostrado 79,1\% del profesorado menor de 45 años; segundo, Edad2[0,33-0,667] ha concentrado el $53,8 \%$ del profesorado entre 45 y 60 años; tercero, Edad3 [0,00-0,333] ha presentado el $80,9 \%$ del profesorado con edad superior a 60 años; cuarto, MediaEdadProfesorado[0,33-0,667) ha concentrado el 45,52\%; quinto, Exper.Docente[0,33-0,667) ha agrupado el $58,1 \%$ de datos relacionados con la variable experiencia docente; por último, la categoría TitulaciónDocente[0-0,333) ha concentrado el 51,53\% de datos relacionados con la titulación académica del docente. En el flujo de datos se constata la tendencia y distribución del profesorado a lo largo de los grados académicos del alumnado, por ende, la variable objetivo "Clase" agrupa el porcentaje del alumnado que ha superado, cambiado o abandonado el grado académico.

Diagrama de Sankey asociado con las características del profesorado

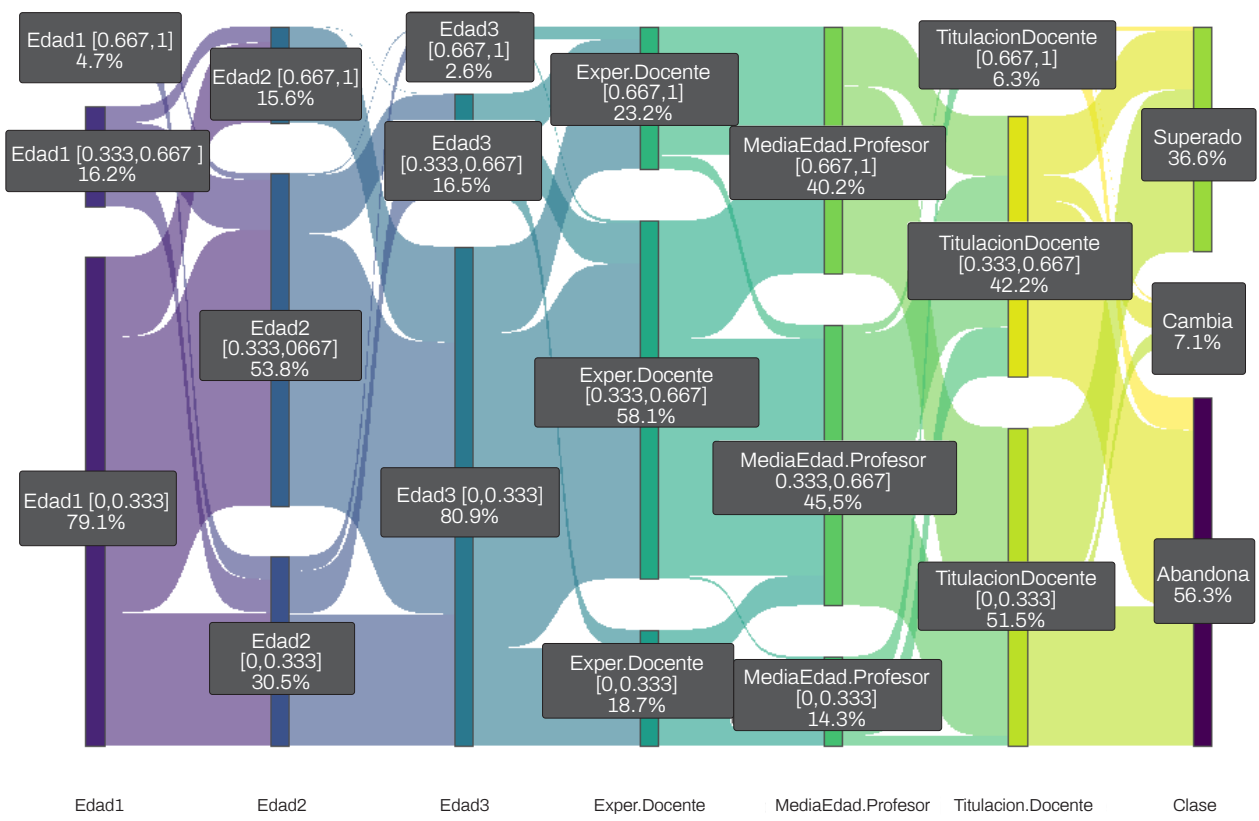

Gráfico 3. Flujo y carga de datos con características del profesorado. Las variables fueron normalizadas en un rango entre 0 y 1 , posteriormente fueron separadas en tres categorías, después, se presenta la carga (\%) de las categorías que facilita la comprensión de lo que ocurre entre las categorías. Al final del gráfico, se muestra la variable denominada "Clase" que representa el estado académico final del alumnado.

Fuente: Elaboración propia 
En la Tabla 3 se muestran las categorías de la variable "Edad3" que representan la cantidad de profesores con edad superior a 60 años, donde el 80,90\% de los datos están concentrados en la categoría "[0-0,33)", es decir, el primer tercio del total profesores. Al mismo tiempo, se ha comprobado dos categorías relevantes: (i) la categoría "[0,33-0,667)" que del total $16,50 \%, 48 \%$ han superado el grado académico y la relación categoría/total fue 1,311; (ii) la categoría "[0,667-1,00]" que del total 2,60\%, $72,30 \%$ han superado el grado académico, y la relación categoría/total fue 1,975. En líneas generales, se demuestra que la participación del tercer tercio del profesorado en el proceso de enseñanza ha influenciado en positivo al estudiantado para superar el grado universitario.

Tabla 3. Resultados de la relación entre las categorías de la variable Edad3 frente a la situación académica del alumno (Superado). La métrica Lift ha destacado los menos significativos cuyo valor es inferior a 1 y los más significativos mayores o iguales a 1 .

\begin{tabular}{|c|c|c|c|c|c|}
\hline \multicolumn{2}{|r|}{ Edad3 } & \multicolumn{4}{|c|}{ Estado académico } \\
\hline & & Abandona & Cambio & Superado & Total \\
\hline \multirow[t]{4}{*}{$0,00-0,33$} & Número de casos & 3188 & 431 & 1791 & 5410 \\
\hline & Frecuencia sobre el total a & $58,90 \%$ & $8,00 \%$ & $33,10 \%$ & $80,90 \%$ \\
\hline & Distribución sobre la categoría & $84,60 \%$ & $90,90 \%$ & $73,20 \%$ & \\
\hline & Relación categoría/total* (Lift) & 1.046 & 1.127 & 0.904 & \\
\hline \multirow[t]{4}{*}{$0,33-0,667$} & Número de casos & 536 & 38 & 529 & 1103 \\
\hline & Frecuencia sobre el total & $48,60 \%$ & $3,40 \%$ & $48,00 \%$ & $16,50 \%$ \\
\hline & Distribución sobre la categoría & $14,20 \%$ & $8,00 \%$ & $21,60 \%$ & \\
\hline & Relación categoría/total (Lift) & 0,863 & 0,479 & 1,311 & \\
\hline \multirow[t]{4}{*}[0,667-1,00]{} & Número de casos & 44 & 5 & 128 & 177 \\
\hline & Frecuencia sobre el total & $24,90 \%$ & $2,80 \%$ & $72,30 \%$ & $2,60 \%$ \\
\hline & Distribución sobre la categoría & $1,20 \%$ & $1,10 \%$ & $5,20 \%$ & \\
\hline & Relación categoría/total (Lift) & 0.442 & 0.394 & 1.975 & \\
\hline Total & & 3768 & 474 & 2448 & 6690 \\
\hline Porcentaje b & & $56,30 \%$ & $7,10 \%$ & $36,60 \%$ & \\
\hline \multicolumn{6}{|c|}{${ }^{*}$ Relación categoría/total = $(\mathrm{a} / \mathrm{b})$} \\
\hline
\end{tabular}


Tabla 4. Resultados de la relación de datos entre la categoría de la variable Edad2 frente al estado académico del alumnado (Superado). Se usa la métrica Lift para destacar los menos significativos cuyo valor es inferior a 1 y los más significativos mayores o iguales a 1.

Edad2

\begin{tabular}{|c|c|}
\hline \multirow[t]{4}{*}{$0,00-0,33$} & Número de casos \\
\hline & Frecuencia sobre el total a \\
\hline & Distribución sobre la categoría \\
\hline & Relación categoría/total* (Lift) \\
\hline \multirow[t]{4}{*}{$0,33-0,667$} & Número de casos \\
\hline & Frecuencia sobre el total \\
\hline & Distribución sobre la categoría \\
\hline & Relación categoría/total (Lift) \\
\hline \multirow[t]{4}{*}[0,667-1,00]{} & Número de casos \\
\hline & Frecuencia sobre el total \\
\hline & Distribución sobre la categoría \\
\hline & Relación categoría/total (Lift) \\
\hline
\end{tabular}

Total

Porcentaje b
Estado académico

Abandona Cambio Superado Total

1446

124

473

2043

\begin{tabular}{rcr}
$70,80 \%$ & $6,10 \%$ & $23,20 \%$ \\
$38,40 \%$ & $26,20 \%$ & $19,30 \%$ \\
\hline 1.258 & 0,859 & 0,6344 \\
\hline 1761 & 256 & 1585
\end{tabular}

3602

$48,90 \% \quad 7,10 \% \quad 44,00 \% \quad 53,80 \%$

$46,70 \% \quad 54,00 \% \quad 64,70 \%$

\begin{tabular}{rrrrr}
\hline 0.869 & 1,000 & 1,202 & \\
5661 & 94 & 390 & 1045 \\
$53,70 \%$ & $9,00 \%$ & $37,30 \%$ & $15,60 \%$ \\
$14,90 \%$ & $19,80 \%$ & $15,90 \%$ & \\
\hline 0,954 & 1,268 & 1,019 & \\
3768 & 474 & 2448 & 6690
\end{tabular}

$56,30 \% \quad 7,10 \% \quad 36,60 \%$

*Relación categoría/total $=(\mathrm{a} / \mathrm{b})$

Fuente: Elaboración propia

Como se aprecia, en la Tabla 4 las categorías de "Edad2", siendo "(0,33-0,667]" la que tuvo una densidad del $53,80 \%$ de los datos. Como consecuencia de haber explorado esta variable, se encontraron dos categorías relevantes: (i) la categoría "[0,33-0,667)" que del total del $53,8 \%$, el $44 \%$ han superado el grado universitario, mientras que, la relación categoría/total fue 1,203. (ii) La categoría "[0,667-1,00]" del total $15,6 \%, 37,30 \%$ han superado el grado académico, y la relación categoría/total de 1,02 . En otras palabras, el segundo y tercer tercio de los profesores entre 45 y 60 años influyeron positivamente en el alumnado para superar el grado universitario. También es interesante observar que en la Tabla 5, están examinadas las categorías de la variable experiencia del profesorado, y que la densidad de los datos fue un $58,10 \%$ en la categoría "[0,33-0,667)". Teniendo en cuenta los datos de esta tabla, se detectan que dos categorías fueron relevantes: (i) la categoría "[0,33-0,667)" que del total $58,10 \%$, el $37,20 \%$ ha superado el grado universitario, y la relación categoría/total fue 1,016; (ii) la categoría "[0,667-1,00]" del total del 23,20\%, el 54,30\% ha superado el grado universitario, y la relación categoría/total fue 1,484. Una importante distinción, que se debe resaltar en la experiencia del profesorado, fue que la segunda y tercera categoría predominó en los estudiantes para superar el grado universitario. 
Tabla 5. Resultados de la relación de datos entre la categoría de la variable experiencia del profesor frente a la situación académica del alumno (Superado). Se utiliza la métrica Lift para destacar los menos significativos cuyo valor es inferior a 1 y los más significativos mayores o iguales a 1.

\begin{tabular}{|c|c|c|c|c|c|}
\hline \multicolumn{2}{|r|}{ Edad2 } & \multicolumn{4}{|c|}{ Estado académico } \\
\hline & & Abandona & Cambio & Superado & Total \\
\hline \multirow[t]{4}{*}{$0,00-0,33$} & Número de casos & 1007 & 84 & 159 & 1250 \\
\hline & Frecuencia sobre el total a & $80,60 \%$ & $6,70 \%$ & $12,70 \%$ & $18,70 \%$ \\
\hline & Distribución sobre la categoría & $26,70 \%$ & $17,70 \%$ & $6,50 \%$ & \\
\hline & Relación categoría/total* (Lift) & 1,432 & 0,944 & 0,347 & \\
\hline \multirow[t]{4}{*}{$0,33-0,667$} & Número de casos & 2134 & 307 & 1445 & 3886 \\
\hline & Frecuencia sobre el total & $54,90 \%$ & $7,90 \%$ & $37,20 \%$ & $58,10 \%$ \\
\hline & Distribución sobre la categoría & $56,60 \%$ & $64,80 \%$ & $59,00 \%$ & \\
\hline & Relación categoría/total (Lift) & 0,975 & 1,113 & 1,016 & \\
\hline \multirow[t]{4}{*}[0,667-1,00]{} & Número de casos & 627 & 83 & 844 & 1554 \\
\hline & Frecuencia sobre el total & $40,30 \%$ & $5,30 \%$ & $54,30 \%$ & $23,20 \%$ \\
\hline & Distribución sobre la categoría & $16,60 \%$ & $17,50 \%$ & $34,50 \%$ & \\
\hline & Relación categoría/total (Lift) & 0,716 & 0,746 & 1,484 & \\
\hline Total & & 3768 & 474 & 2448 & 6690 \\
\hline Porcentaje b & & $56,30 \%$ & $7,10 \%$ & $36,60 \%$ & \\
\hline
\end{tabular}

A la luz de los resultados presentados en las Tablas 3, 4 y 5, se puede observar que la "relación categoría/total (Lift)" ha conseguido captar categorías significativas en las variables del profesorado. La transmisión consolidada y comprensible de los resultados se reflejan con claridad en un mapa de calor (Gráfico 4), destacando las etiquetas con valor superior a 1 , mostradas con colores más oscuros (rojizos). En concreto, la etiqueta Edad3 [0,667-1,00] con 1,976 en el estado académico Superado ha sido altamente significativa para los datos analizados. De manera general, tanto el segundo y tercer tercio de las variables experiencia docente, media de edad, Edad2 y Edad3 han influido en la superación o cambio del grado universitario. Es decir, el encauzamiento del éxito académico del estudiantado se potencia cuando la participación de este grupo de variables es superior o igual a dos tercios. Por otra parte, el Gráfico 5 presenta la incidencia que tuvo el profesorado experimentado (Edad3) frente al estado académico final del estudiantado. De acuerdo con los resultados obtenidos se constata que cuanto mayor es la participación de ellos en el proceso de enseñanza, el alumnado tuvo mayores casos de éxito académico. 


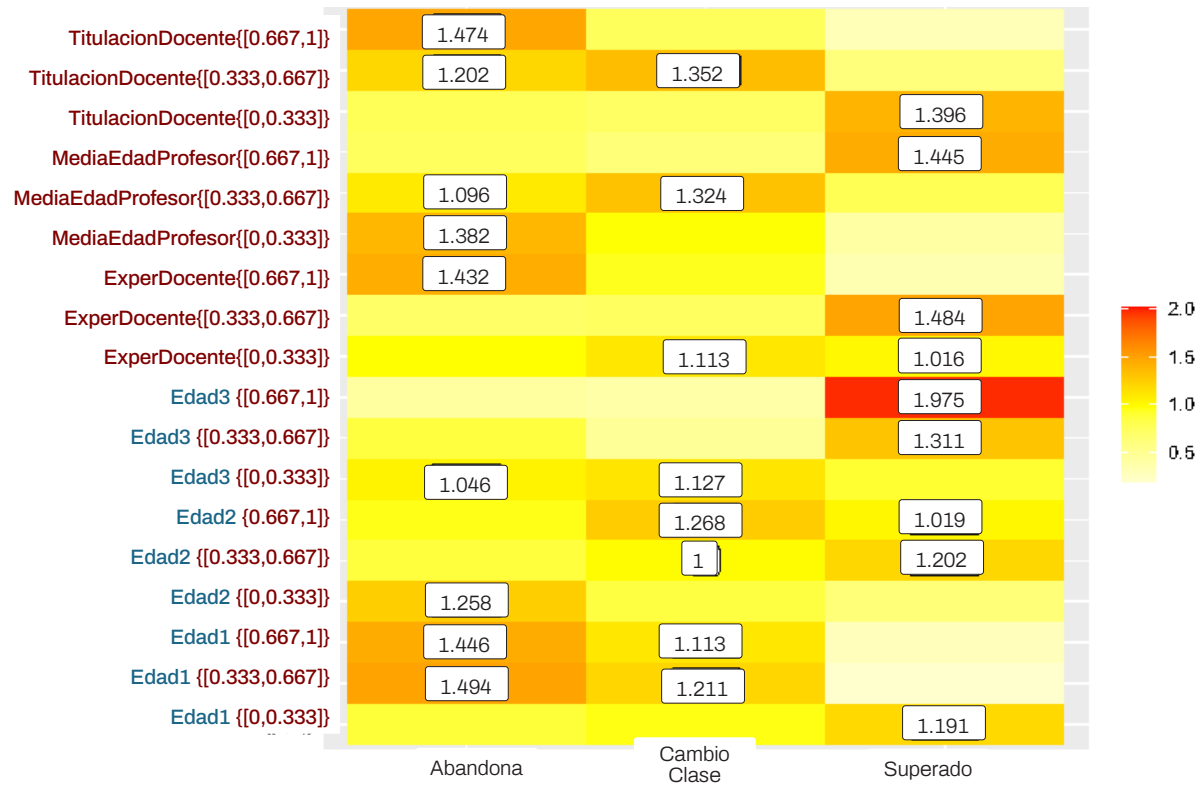

Gráfico 4. Mapa de calor con las categorías variables y el estado académico del alumnado, el color más oscuro (rojizo) indica una alta asociación entre la categoría y la superación del grado universitario. La mayor concentración de asociaciones se ha dado en el segundo y tercer tercio de las variables categorizadas. La categoría Edad3 [0,667-1,00] muestra una alta asociación. Fuente: Elaboración propia

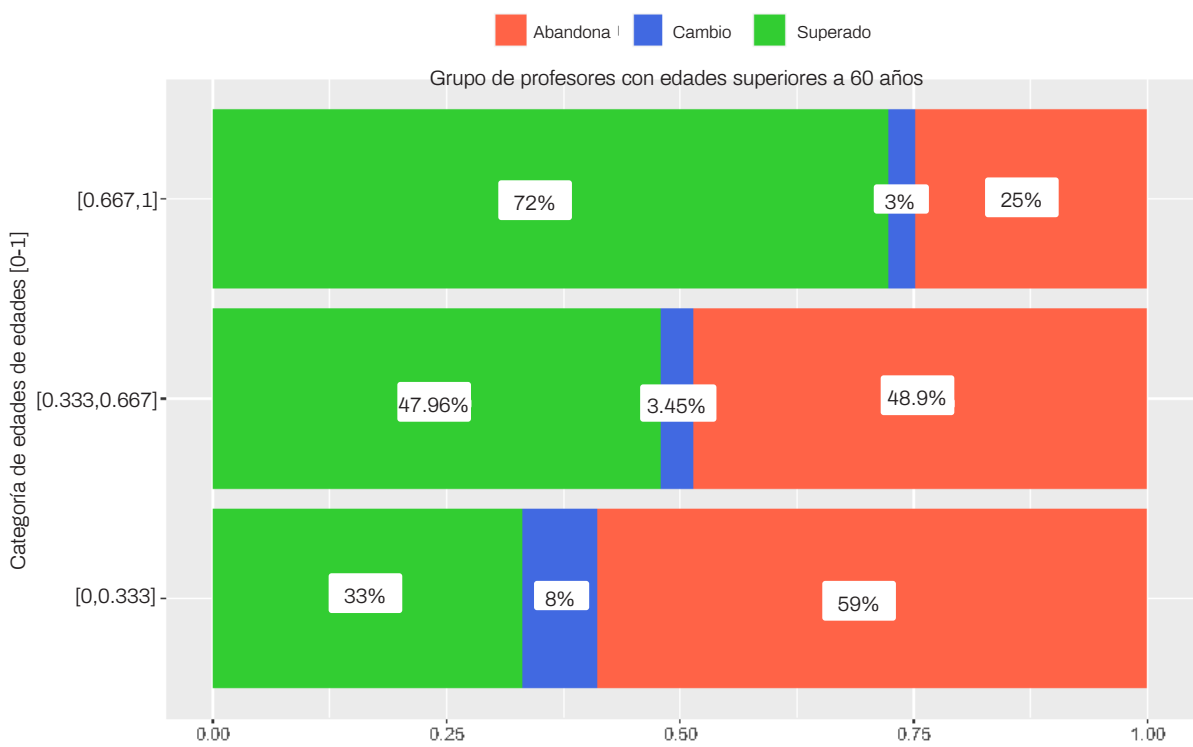

Gráfico 5. Proporción de participación del profesorado experimentado en el proceso de enseñanza del alumnado y el estado académico del alumnado, cada categoría contiene tres barras horizontales que van de izquierda a derecha. La primera relacionada con los que han superado el grado académico, la segunda de los que han cambiado de grado y la tercera de los que han abandonado. La mayor proporción del alumnado que ha superado el grado académico estuvo centrada en docentes con participación superior al 66\%, concretamente la categoría [0.66-1].

Fuente: Elaboración propia 


\section{Discusión y conclusiones}

En respuesta a las preguntas de investigación planteadas al inicio de este estudio, podemos decir, que el proceso de enseñanza en las titulaciones universitarias es eficaz cuando el número total de profesores que imparten docencia en el primer curso, se distribuye proporcionalmente entre el segundo y el tercer tercio, tanto en lo que se refiere a profesores con experiencia y madurez académica, como a grupos de edad. Existen estudios análogos como se muestra en la bibliografía citada, sin embargo, esta investigación se ha centrado en demostrar y facilitar la comprensión de los resultados relevantes sobre los factores y vínculos significativos que llevan a los estudiantes a superar la titulación universitaria.

Si bien es cierto, la dificultad de encontrar una distribución universal de los docentes para guiar a los estudiantes hacia el éxito académico es compleja. La disposición de una estrategia flexible y ajustada a la IES es una ruta alternativa. De hecho, Trigwell et al. (1999) matizan la importancia de trabajar con el personal académico para fomentar la adopción de enfoques de mayor calidad en la enseñanza. Al mismo tiempo, Gutiérrez et al. (2018) dicen que este ambiente promueve confianza y que, además, consolida la positividad y motiva al alumnado a un mayor compromiso académico para completar la educación sin abandonar los estudios.

Respecto a la experiencia del profesorado, nuestros resultados coinciden con Pascarella et al. (1996) que entre los hallazgos encontrados han sugerido que la práctica docente eficaz influye positivamente en el aprendizaje, además de incrementar el número de alumnos con éxito académico. De hecho, Roksa y Whitley (2017) afirman que la madurez y el tipo de enseñanza del docente, a través de la iteración alumnoprofesor ha contribuido como un precursor en el alumnado para superar el grado universitario. Por otra parte, Boluda y López (2012) en su investigación expresan que la "calidad" del profesorado es un poderoso predictor que se relaciona directamente con el rendimiento del alumnado y es, posiblemente, uno de los componentes más decisivos de cualquier proceso formativo. De igual forma, el éxito académico no solo está ligado a las actividades y cualidades de los profesores, sino también a la calidad del esfuerzo realizado por el alumno (Valadas et al., 2017). A pesar de estos hallazgos, los resultados de este estudio deben interpretarse con cierta cautela, ya que los datos solo representan a una institución y los resultados de este estudio pueden no ser trasladables a otras universidades.

En cuanto a las edades del profesorado se establece una idea clara, pero conviene distinguir entre profesores experimentados y noveles. Dado que la edad, como tal, puede ser discriminatoria si no se contextualiza adecuadamente. Esta distinción se aproxima al estudio de Fogarty et al. (1983), donde se indica que el profesorado experimentado tiene en cuenta una mayor variedad de objetivos e instrucciones para la toma de decisiones en el aula; curiosamente, lo contrario ocurre con los profesores noveles. Pero, sin embargo, los novatos son más propensos en detectar signos de rendimiento académico del alumnado que los profesores experimentados. Dicho esto, y en consonancia con los resultados, los profesores maduros (Edad2) y experimentados (Edad3) fueron eficaces en tutelar la superación del grado académico del alumnado, siempre que su participación comprendiera el segundo y tercer tercio del 
total de los profesores que impartieron clase. En términos generales, el profesorado con madurez y experiencia educativa generan un entorno académico fiable y positivo para los alumnos.

Como se ha observado, los resultados obtenidos en este estudio consideramos que son de una importancia relevante. Ya que las razones para creer que las implicaciones asociadas al éxito académico del alumnado, no solo deben ajustarse al contexto, política y normas universitarias; sino, que además se sugiere la distribución del profesorado asignado a impartir clases en el primer curso. Más concretamente, se proponen dos alternativas significativas basadas en los resultados: La primera, está relacionada con que la edad del profesorado experimentado y maduro tenga una tasa de participación superior al 33\%. Todo ello, con el fin de retener al alumno y descartar la deserción universitaria; y, la segunda, sugerir que los profesores con un nivel de madurez en la enseñanza superior al segundo y tercer tercio del total de profesores participe en el proceso de enseñanza.

Desde otra perspectiva, el estudio adolece de las características socioeconómicas y del perfil de los estudiantes, que son temas también de interés para profundizar en el análisis del éxito académico. Ambas se consideran limitaciones de este trabajo. De hecho, el estudio de Roksa y Kinsley (2019) indica que se alcanza una mayor posibilidad de éxito académico en estudiantes con mayores recursos. A su vez, Van Herpen et al. (2017) han examinado el perfil de los estudiantes centrados en la autoeficacia debido a la correspondencia con las características del profesorado. Como estudio posterior, se podría examinar el factor familiar y socio económico del alumnado, ya que el refuerzo con programas extracurriculares, acceso a recursos y ayudas estimulan la superación del grado universitario y, a su vez, permitirá al centro universitario impulsar el encauzamiento del estudiante hacia el éxito académico.

\section{Agradecimientos}

Queremos expresar nuestro profundo agradecimiento a las autoridades de la IES por permitir el acceso, la exploración y el análisis de la información. Especialmente al Departamento de Planificación Académica. Esta investigación ha sido apoyada por el MINECO/FEDER en el marco del Proyecto Nacional de Investigación TIN2017-89517-P

\section{Referencias}

Abarca, M. S., Gómez, M. T. P. y Covarrubias, M. de L. V. (2015). Análisis de los factores que contribuyen al éxito académico en estudiantes universitarios. Revista Internacional de Educación y Aprendizaje, 3(2), 124-137.

Aleksandrova, Y. y Parusheva, S. (2019). Social media usage patterns in higher education institutions - An empirical study. International Journal of Emerging Technologies in Learning, 14(5), 108-121. https://doi.org/10.3991/ijet.v14i05.9720

Alyahyan, E. y Düştegör, D. (2020). Predicting academic success in higher education: literature review and best practices. In International Journal of Educational Technology in Higher Education, 17(1), 1-21. https://doi.org/10.1186/s41239-020-0177-7

Amida, A., Algarni, S. y Stupnisky, R. (2020). Testing the relationships of motivation, time management and career aspirations on graduate students' academic success. Journal of Applied Research in Higher Education. https://doi.org/10.1108/JARHE-04-2020-0106 
Angervall, P. (2018). The academic career: a study of subjectivity, gender and movement among women university lecturers. Gender and Education, 30(1), 105-118. https://doi.org/10.1 $\underline{080 / 09540253.2016 .1184234}$

Araque, F., Roldán, C. y Salguero, A. (2009). Factors influencing university drop out rates. Computers and Education, 53(3), 563-574. https://doi.org/10.1016/j.compedu.2009.03.013

Boluda, I. K. y López, N. V. (2012). El docente universitario y sus efectos en el estudiante. Estudios Sobre Educacion, 23(23), 157-182. https://bit.ly/3rnOjsG

Breiman, L. (2001). Random Forests. Machine Learning, 45(1), 5-32. https://doi. org/10.1023/A:1010933404324

Brin, S., Motwani, R., Ullman, J. D. y Tsur, S. (1997). Dynamic itemset counting and implication rules for market basket data. ACM SIGMOD Record, 26(2), 255-264. https://doi. org/10.1145/253262.253325

Campbell, C. M., Smith, M., Dugan, J. P. y Komives, S. R. (2012). Mentors and college student leadership outcomes: The importance of position and process. Review of Higher Education, 35(4), 595-625. https://doi.org/10.1353/rhe.2012.0037

Campbell, H. E., Steiner, S. y Gerdes, K. (2005). Student Evaluations of Teaching: How You Teach and Who You Are. Journal of Public Affairs Education, 11(3), 211-231. https://doi.org/10. 1080/15236803.2005.12001395

Chickering, A. W. y Gamson, Z. F. (1987). Seven Principles for Good Practice in Undergraduate Graduation. AAHE Bulletin;, 39(7), 3-7.

Cho, Y., Kim, M., Svinicki, M. D. y Decker, M. L. (2011). Exploring teaching concerns and characteristics of graduate teaching assistants. Teaching in Higher Education, 16(3), 267-279. https:// doi.org/10.1080/13562517.2010.524920

Crisp, G., Taggart, A. y Nora, A. (2015). Undergraduate Latina/o Students: A Systematic Review of Research Identifying Factors Contributing to Academic Success Outcomes. Review of Educational Research, 85(2), 249-274. https://doi.org/10.3102/0034654314551064

Cunha, J. M., Miller, T. y Weisburst, E. (2018). Information and College Decisions: Evidence From the Texas GO Center Project. Educational Evaluation and Policy Analysis, 40(1), 151-170. https://doi.org/10.3102/0162373717739349

Darling-Hammond, L. (2000). Teacher Quality and Student Achievement. Education Policy Analysis Archives, 8(0), 1. https://doi.org/10.14507/epaa.v8n1.2000

de Boer, H., Donker, A. S. y Van der Werf, M. P. C. (2014). Effects of the Attributes of Educational Interventions on Students'Academic Performance:AMeta-Analysis. Review of Educational Research, 84(4), 509-545. https://doi.org/10.3102/0034654314540006

Fogarty, J. L., Wang, M. C. y Creek, R. (1983). A Descriptive Study of Experienced and Novice Teachers' Interactive Instructional Thoughts and Actions, The Journal of Educational Research, 77, 22-32. https://doi.org/10.2307/27540012

Glogowska, M., Young, P. y Lockyer, L. (2007). Should I go or should I stay? Active Learning in Higher Education, 8(1), 63-77. https://doi.org/10.1177/1469787407074115

Gutiérrez, M., Tomás, J.-M. y Alberola, S. (2018). Apoyo docente, compromiso académico y satisfacción del alumnado universitario. Estudios Sobre Educación, 35(0), 535-555. https://doi. org/10.15581/004.35.535-555

Guanin-Fajardo, J., Casillas, J. y Chiriboga-Casanova, W. (2019). Aprendizaje semi-supervisado para descubrir la escala de tiempo promedio de graduación de estudiantes universitarios. Revista Conrado, 15(70). Recuperado de https://bit.ly/3rxdnfe

Jacobi, M. (1991). Mentoring and Undergraduate Academic Success: A Literature Review. Review of Educational Research, 61(4), 505-532. https://doi.org/10.3102/00346543061004505

Jepsen, C. (2005). Teacher characteristics and student achievement: Evidence from teacher surveys. Journal of Urban Economics, 57(2), 302-319. https://doi.org/10.1016/j.jue.2004.11.001

Kara, N., Çubukçuoğlu, B. y Elçi, A. (2020). Using social media to support teaching and learning in higher education: An analysis of personal narratives. Research in Learning Technology, 28, 1-16. https://doi.org/10.25304/rlt.v28.2410 
Konrad, T., Wiek, A. y Barth, M. (2021). Learning processes for interpersonal competence development in project-based sustainability courses - insights from a comparative international study. International Journal of Sustainability in Higher Education, ahead-of-p(ahead-of-print). https://doi.org/10.1108/ijshe-07-2020-0231

Korhonen, V. y Törmä, S. (2016). Engagement with a teaching career - how a group of finnish university teachers experience teacher identity and professional growth. Journal of Further and Higher Education, 40(1), 65-82. https://doi.org/10.1080/0309877X.2014.895301

Korobova, N. y Starobin, S. S. (2015). A comparative study of student engagement, satisfaction, and academic success among international and american students. Journal of International Students, 5(1), 72-85. http://jistudents.org

Le, T., Bolt, D., Camburn, E., Goff, P. y Rohe, K. (2017). Latent Factors in Student-Teacher Interaction Factor Analysis. Journal of Educational and Behavioral Statistics, 42(2), 115-144. https:// doi.org/10.3102/1076998616676407

Leal Filho, W., Shiel, C. y Paço, A. (2016). Implementing and operationalising integrative approaches to sustainability in higher education: the role of project-oriented learning. Journal of Cleaner Production, 133, 126-135. https://doi.org/10.1016/j.jclepro.2016.05.079

Livengood, J. M. (1992). Students' motivational goals and beliefs about effort and ability as they relate to college academic success. Research in Higher Education, 33(2), 247-261. https://doi. org/10.1007/BF00973581

Lizzio, A., Wilson, K. y Simons, R. (2002). University students' perceptions of the learning environment and academic outcomes: Implications for theory and practice. Studies in Higher Education, 27(1), 27-52. https://doi.org/10.1080/03075070120099359

Marginson, S. (2014). Higher education and public good. In Thinking About Higher Education (Vol. 9783319032, Issue 4, pp. 53-69). Wiley/Blackwell (10.1111). https://doi.org/10.1007/9783-319-03254-2-5

Mishra, B. K. y Sahoo, A. K. (2016). Evaluation of faculty performance in education system using classification technique in opinion mining based on GPU, Advances in Intelligent Systems and Computing, 411, 109-119. https://doi.org/10.1007/978-81-322-2731-1_10

Mishra, S. (2020). Social networks, social capital, social support and academic success in higher education: A systematic review with a special focus on 'underrepresented' students. Educational Research Review, 29, 100307. https://doi.org/10.1016/i.edurev.2019.100307

Nagda, B. A., Gregerman, S. R., Jonides, J., Von Hippel, W. y Lerner, J. S. (1998). Undergraduate student-faculty research partnerships affect student retention. In Review of HigherEducation, 22(1), 55-72. https://doi.org/10.1353/rhe.1998.0016

Nasser-Abu Alhija, F. (2017). Teaching in higher education: Good teaching through students' lens. Studies in Educational Evaluation, 54, 4-12. https://doi.org/10.1016/j.stueduc.2016.10.006

Pascarella, E. T., Edison, M., Hagedorn, L. S., Nora, A. y Terenzini, P. T. (1996). Influences on students' internal locus of attribution for academic success in the first year of college. Research in Higher Education, 37(6), 731-756. https://doi.org/10.1007/BF01792954

Pineda, C., Bermúdez, J.-J., Rubiano, Á., Pava, N., Suárez, R. y Cruz, F. (2014). Student engagement and academic performance in the colombian university context. RELIEVE - Revista Electronica de Investigacion y Evaluacion Educativa, 20(2), 1-19. https://doi.org/10.7203/ relieve.20.2.4238

R CoreTeam, D. C. (2019). A Language and Environment for Statistical Computing. In R Foundation for Statistical Computing (Vol. 739, Issue 09/18/2009, p. ISBN 3-900051-07-0-ISBN 3-900051-07-0). https://doi.org/10.1007/978-3-540-74686-7

Respondek, L., Seufert, T., Stupnisky, R. y Nett, U. E. (2017). Perceived academic control and academic emotions predict undergraduate university student success: Examining effects on dropout intention and achievement. Frontiers in Psychology, 8(MAR), 243. https://doi. org/10.3389/fpsyg.2017.00243

Roksa, J. y Whitley, S. E. (2017). Fostering Academic Success of First-Year Students: Exploring the Roles of Motivation, Race, and Faculty. Journal of College Student Development, 58(3), 333-348. https://doi.org/10.1353/csd.2017.0026 
Romanski, P. y Kotthoff, L. (2016). FSelector: Selecting Attributes. https://cran.r-project.org/ package $=$ FSelector

Salminen-Tuomaala, M. y Koskela, T. (2020). How can simulation help with learning project work skills? Experiences from higher education in Finland. Educational Research, 62(1), 77-94. https:// doi.org/10.1080/00131881.2020.1711791

Sanvitha, K. T., Liyanage, S. R. y Bhatt, C. M. (2018). A data mining approach to identify the factors affecting the academic success of tertiary students in sri lanka. Lecture Notes on Data Engineering and Communications Technologies, 11, 179-197. https://doi.org/10.1007/9783-319-68318-8_9

Shetu, S. F., Saifuzzaman, M., Moon, N. N., Sultana, S. y Yousuf, R. (2021). Student's performance prediction using data mining technique depending on overall academic status and environmental attributes. Advances in Intelligent Systems and Computing, 1166, 757-769. https://doi.org/10.1007/978-981-15-5148-2_66

Souchon, N., Kermarec, G., Trouilloud, D. y Bardin, B. (2020). Influence of teachers' political orientation and values on their success prediction toward students from different socioeconomic background. Revue Europeenne de Psychologie Appliquee, 70(5), 100553. https://doi. org/10.1016/i.erap.2020.100553

Struyven, K., Dochy, F. y Janssens, S. (2003). Students' Perceptions about New Modes of Assessment in Higher Education: A Review BT - Optimising new modes of assessment: In search of qualities and standards. Optimising New Modes of Assessment: In Search of Qualities and Standards, 1(Chapter 8), 171-223. https://doi.org/10.1007/0-306-48125-1_8

Tinto, V. (1975). Dropout from Higher Education: A Theoretical Synthesis of Recent Research. Review of Educational Research, 45(1), 89-125. https://doi.org/10.3102/00346543045001089

Trigwell, K., Prosser, M. y Waterhouse, F. (1999). Relations between teachers' approaches to teaching and students' approaches to learning. Higher Education, 37(1), 57-70. https:// doi.org/10.1023/A:1003548313194

Trolian, T. L., Jach, E. A. y Archibald, G. C. (2021). Shaping Students' Career Attitudes toward Professional Success: Examining the Role of Student-Faculty Interactions. Innovative Higher Education, 46(2), 111-131. https://doi.org/10.1007/s10755-020-09529-3

Valadas, S. T., Almeida, L. S. y Araújo, A. M. (2017). The Mediating Effects of Approaches to Learning on the Academic Success of First-Year College Students. Scandinavian Journal of Educational Research, 61(6), 721-734. https://doi.org/10.1080/00313831.2016.1188146

Van den Berg, M. N. y Hofman, W. H. A. (2005). Student success in university education: A multimeasurement study of the impact of student and faculty factors on study progress. Higher Education, 50(3), 413-446. https://doi.org/10.1007/s10734-004-6361-1

Van Herpen, S. G. A., Meeuwisse, M., Hofman, W. H. A., Severiens, S. E. y Arends, L. R. (2017). Early predictors of first-year academic success at university: pre-university effort, pre-university self-efficacy, and pre-university reasons for attending university. Educational Research and Evaluation, 23(1-2), 52-72. https://doi.org/10.1080/13803611.2017.1301261

Vo, T. N. C., Nguyen, H. P. y Vo, T. N. T. (2016). Making kernel-based vector quantization robust and effective for incomplete educational data clustering. Vietnam Journal of Computer Science, 3(2), 93. https://doi.org/10.1007/s40595-016-0060-6

Walder, A. M. (2017). Pedagogical Innovation in Canadian higher education: Professors' perspectives on its effects on teaching and learning. Studies in Educational Evaluation, 54, 71-82. https://doi.org/10.1016/j.stueduc.2016.11.001

Winterer, E. R., Froyd, J. E., Borrego, M., Martin, J. P. y Foster, M. (2020). Factors influencing the academic success of Latinx students matriculating at 2-year and transferring to 4-year US institutions-implications for STEM majors: asystematic review of the literature. In International Journal of STEM Education 7(1), 34. https://doi.org/10.1186/s40594-020-00215-6

Young, P., Glogowska, M. y Lockyer, L. (2007). Conceptions of early leaving: A comparison of the views of teaching staff and students. Active Learning in Higher Education, 8(3), 275-287. https://doi.org/10.1177/1469787407081882 


\section{Anexo I}

Descripción de las variables usadas para el estudio

\begin{tabular}{|c|c|c|c|}
\hline Variables & Descripción & Tipo & Rango de datos \\
\hline Facultad & Nombre de la Facultad & Categórica & 5 Facultades \\
\hline GradoUniversitario & $\begin{array}{l}\text { Titulación académica en la que se ha } \\
\text { matriculado el estudiante }\end{array}$ & Categórica & 22 titulaciones \\
\hline Edad1 & $\begin{array}{l}\text { Cantidad de profesores menores } \\
\text { de } 45 \text { años }\end{array}$ & Numérica & $0-15$ \\
\hline Edad2 & $\begin{array}{l}\text { Cantidad de profesores entre } 46 \text { y } \\
60 \text { años }\end{array}$ & Numérica & $0-15$ \\
\hline Edad3 & $\begin{array}{l}\text { Cantidad de profesores mayores } \\
\text { de } 61 \text { años }\end{array}$ & Numérica & $0-15$ \\
\hline $\begin{array}{l}\text { ExperienciaDo- } \\
\text { cente }\end{array}$ & $\begin{array}{l}\text { Media de experiencia docente del } \\
\text { profesorado (años) }\end{array}$ & Numérica & 4-35 \\
\hline $\begin{array}{l}\text { MediaEdadProfe- } \\
\text { sor }\end{array}$ & Media de edad del profesorado & Numérica & $35-75$ \\
\hline TitulaciónDocente & $\begin{array}{l}\text { Ponderación del profesorado que } \\
\text { dictó clases en primer curso }\end{array}$ & Numérica & $0-1$ \\
\hline MediaNotas & $\begin{array}{l}\text { Media de notas del primer curso del } \\
\text { estudiante }\end{array}$ & Numérica & $0-10$ \\
\hline MediaAsistencia & $\begin{array}{l}\text { Media de asistencia a clases del } \\
\text { estudiante }\end{array}$ & Numérica & $0-100$ \\
\hline TiempoSupera & $\begin{array}{l}\text { Cantidad de cursos matriculado para } \\
\text { superar el primer año }\end{array}$ & Numérica & $0-5$ \\
\hline TasaSuperacion & $\begin{array}{l}\text { Ponderación de convocatorias a } \\
\text { exámenes }\end{array}$ & Numérica & $0-3$ \\
\hline CuentaGrados & $\begin{array}{l}\text { Cantidad de grados matriculado en } \\
\text { primer curso }\end{array}$ & Numérica & $1-5$ \\
\hline CambioGrado & $\begin{array}{l}\text { Indicador para conocer si el grado } \\
\text { académico es el inicial o ha cambiado } \\
\text { de grado. }\end{array}$ & Numérica & $0-1$ \\
\hline Clase & $\begin{array}{l}\text { Estado académico del estudiante al } \\
\text { finalizar el grado académico }\end{array}$ & Categórica & $\begin{array}{c}\text { Abandona, } \\
\text { Cambio, Superado }\end{array}$ \\
\hline
\end{tabular}

\section{Cómo citar en APA:}

Guanin-Fajardo, J. H. y Casillas J. (2022). Contexto universitario, profesores y estudiantes: vínculos y éxito académico. Revista Iberoamericana de Educación, 88(1), 127-146. https://doi.org/10.35362/rie8814733 